\title{
Telomere length predicts all-cause mortality in patients with type 1 diabetes
}

\author{
A. S. Astrup • L. Tarnow • A. Jorsal • M. Lajer • \\ R. Nzietchueng $\cdot$ A. Benetos $\cdot$ P. Rossing $\cdot$ H.-H. Parving
}

Received: 17 June 2009 / Accepted: 2 September 2009/Published online: 4 October 2009

(C) Springer-Verlag 2009

\begin{abstract}
Aims/hypothesis Type 1 diabetic patients with diabetic nephropathy have increased mortality and morbidity compared with normoalbuminuric patients. Telomere length in proliferative cells is inversely related to the total number of cell divisions, and therefore to biological age. We aimed to evaluate differences in telomere length in patients with type 1 diabetes with or without diabetic nephropathy; we also evaluated the prognostic value of telomere length.

Methods In a prospective follow-up study, 157 type 1 diabetic patients with diabetic nephropathy and a control group of 116 patients with type 1 diabetes and normoalbuminuria were followed for 11.1 years (range 0.2-12.9). Telomere length was measured from DNA samples extracted from white blood cells at baseline.

Results The mean telomere length did not differ between patients with or without diabetic nephropathy, and was similar in men and women, but was inversely correlated with age and systolic blood pressure, $p<0.05$. When dividing patients into tertiles after telomere length, 36
\end{abstract}

A. S. Astrup $(\bowtie) \cdot$ L. Tarnow $\cdot$ A. Jorsal $\cdot$ M. Lajer $\cdot$ P. Rossing

Steno Diabetes Center, Att Ingelise Holstein,

Niels Steensens Vej 1,

2820 Gentofte, Denmark

e-mail: ansa@steno.dk

R. Nzietchueng $\cdot$ A. Benetos

Geriatric Department and INSERM U691, University of Nancy,

Nancy, France

H.-H. Parving

Department of Endocrinology, Rigshospitalet,

Copenhagen, Denmark

H.-H. Parving

Faculty of Health, Aarhus University,

Aarhus, Denmark
(37\%) patients died in the tertile with the shortest telomere length, $24(28 \%)$ died in the middle tertile, and $15(17 \%)$ of patients in the tertile with the longest telomere length died, $\log$ rank test $p=0.017$. After adjustment for traditional risk factors, telomere length was still predictive of all-cause mortality.

Conclusions/interpretation In patients with type 1 diabetes we found no differences in telomere length between patients with or without diabetic nephropathy. We also found that telomere length was associated with all-cause mortality; however, confirmative studies are needed to verify our findings.

Keywords Diabetic nephropathy · Mortality · Telomere length · Type 1 diabetes

\section{Introduction}

Telomeres are repetitive DNA sequences located at the end of the chromosomes. During replication the length of the telomeres shorten, and thus in proliferative somatic cells the length of telomeres is inversely related to the total number of cell divisions, and therefore to biological age [1].

Inflammation increases the loss of telomere length in cultured proliferative cells [2]. Thus in theory, telomere length is influenced by the number of replications and inflammation. Patients with type 1 diabetes and diabetic nephropathy have an increased mortality compared with patients without diabetic nephropathy. If short telomere length is a measure of biological age and of inflammatory burden, then telomere length might be of value in patients with diabetic nephropathy by pointing out individuals at high risk. In the present study we aimed to examine differences in telomere length between patients with or 
without diabetic nephropathy. We also evaluated the prognostic value of telomere length with regards to allcause mortality, end-stage renal disease and progression of renal disease.

\section{Methods}

During 1993, we invited all albuminuric patients $(n=242)$ attending Steno Diabetes Center in Gentofte, Denmark who had type 1 diabetes and diabetic nephropathy and had their glomerular filtration rate measured the same year to participate in our study. In total, 198 patients accepted. Diabetic nephropathy was diagnosed using the following criteria: persistent albuminuria $\geq 300 \mathrm{mg} / 24 \mathrm{~h}$ in two of three consecutive $24 \mathrm{~h}$ urine collections, presence of retinopathy and no evidence of other renal or urinary tract disease [3]. As control individuals, we recruited 192 patients with longstanding type 1 diabetes and persistent normoalbuminuria (urinary albumin excretion rate $<30 \mathrm{mg} / 24 \mathrm{~h}$ ). The two groups were matched for sex, age and duration of diabetes [4].

Telomere length was measured in 157 patients with diabetic nephropathy and 116 patients with persistent normoalbuminuria. Sufficient sample material was not available from all patients. Patients who had telomere length measured did not differ from patients who did not have telomere length measured with regards to clinical, laboratory and demographic data.

Patients were followed until 1 September 2006 or until death. Two masked observers reviewed all death certificates independently.

The study was approved by the local ethics committee and all patients gave their informed consent.

For measurement of telomere length, DNA samples were digested overnight with restriction enzymes HinfI (40 U) and RsaI (40 U) (Roche, Basel, Switzerland). Twenty-four DNA samples $(1.5 \mu \mathrm{g}$ each $)$ and five DNA ladders $(1 \mathrm{~kb}$ DNA ladder plus $\gamma$ DNA/HindIII fragments; Roche) were resolved on an $0.8 \%$ agarose gel $(15 \mathrm{~cm} \times 25 \mathrm{~cm})$ at $40 \mathrm{~V}$ (Bio-Rad, Marnes-la-Coquette, France). After $20 \mathrm{~h}$, the DNA was depurinated for $30 \mathrm{~min}$ in $0.25 \mathrm{mmol} / \mathrm{l} \mathrm{HCl}$, denatured for $30 \mathrm{~min}$ in $0.5 \mathrm{~mol} / \mathrm{l} \mathrm{NaOH}-1.5 \mathrm{~mol} / \mathrm{l} \mathrm{NaCl}$ and neutralised for $30 \mathrm{~min}$ in $0.5 \mathrm{~mol} / 1$ Tris, $\mathrm{pH} 8$ $1.5 \mathrm{~mol} / \mathrm{l} \mathrm{NaCl}$. The DNA was transferred for $1.5 \mathrm{~h}$ to a positively charged nylon membrane using a vacuum blotter (Bio-Rad). The membranes were hybridised at $65^{\circ} \mathrm{C}$ with the telomeric probe [digoxigenin 3'-end labelled 5'$\left.(\mathrm{CCTAAA})_{3}\right]$ overnight in $5 \times \mathrm{SSC}, 0.1 \%$ Sarkosyl, $0.02 \%$ SDS and $1 \%$ blocking reagent (Roche). The membranes were washed three times at room temperature in $2 \times \mathrm{SSC}$, $0.1 \%$ SDS each for $15 \mathrm{~min}$ and once in $2 \times \mathrm{SSC}$ for $15 \mathrm{~min}$. The digoxigenin-labelled probe was detected by the digoxigenin luminescent detection procedure (Roche) and exposed on x-ray film. Each DNA sample was measured in duplicate.

Statistical analysis At baseline, urinary albumin excretion rate and serum creatinine were non-normally distributed and therefore $\log$ transformed before analysis and given as medians (range). All other values are given as means \pm SD. For normally distributed variables, comparison between groups was performed by an unpaired Student's $t$ test or analysis of variance (ANOVA). For normally and nonnormally distributed continuous variables, a Mann-Whitney $U$ test or Kruskal-Wallis test were used for comparison between groups. A $\chi^{2}$ test was used to compare noncontinuous variables.

Logrank test was used to compare the tertiles of patients divided by telomere length.

First, the prognostic value of telomere length was analysed in unadjusted Cox regression analysis with telomere length both as a continuous variable and a categorical variable. All patients were evaluated as one group but the patients with diabetic nephropathy were also analysed separately. Thereafter the models were adjusted for age, sex, smoking, previous event, log urinary albumin excretion, $\mathrm{HbA}_{1 \mathrm{c}}$, total cholesterol and telomere length.

End-stage renal failure and decline in GFR was only relevant in patients with diabetic nephropathy. Linear regression analysis of serial GFR determinations in each individual was used to estimate the rate of decline in kidney function with time.

Two-tailed $p$ values $\leq 0.05$ were considered significant. All calculations were made using SPSS version 13.0.

\section{Results}

Patients with diabetic nephropathy did not differ significantly compared with normoalbuminuric patients with regards to: sex (male/female) $67 / 49$ vs $89 / 68$; age $41 \pm 9$ vs $43 \pm 10$ years; duration of diabetes $28 \pm 8$ vs $28 \pm 8$ years; BMI $23.9 \pm 3.2$ vs $23.6 \pm 2.4 \mathrm{~kg} / \mathrm{m}^{2}$; and prevalence of smokers $47 \%$ vs $40 \%$. The groups were different with regards to total cholesterol $5.6 \pm 1.2$ vs $4.8 \pm 0.9 \mathrm{mmol} / \mathrm{l}$; systolic blood pressure $150 \pm 22$ vs $131 \pm 17 \mathrm{mmHg}$; diastolic blood pressure $86 \pm 13$ vs $75 \pm 10 \mathrm{mmHg}$; and $\mathrm{HbA}_{1 \mathrm{c}} 9.7 \pm 1.6 \%$ vs $8.5 \pm 1.1 \%$. The mean GFR in patients with nephropathy was $75 \pm 32$, and the rate of decline in GFR during follow-up was $4.2 \pm 4.1 \mathrm{ml} / \mathrm{min} /$ year. Follow-up time was 11.1 years $(0.2-12.9)$.

Telomere length did not differ between patients with or without diabetic nephropathy $(7.2 \pm 0.8 \mathrm{~kb}$ vs $7.2 \pm 0.8 \mathrm{~kb}$, respectively $[p=0.9])$, nor between men and women, $p=$ 
Table 1 Cox regression analysis with telomere length (continuous variable and categorical variable) as predictor of all-cause mortality

\begin{tabular}{|c|c|c|c|c|}
\hline \multirow[t]{3}{*}{ Variable } & \multicolumn{4}{|l|}{ All-cause mortality } \\
\hline & \multicolumn{2}{|c|}{ Unadjusted analysis } & \multicolumn{2}{|c|}{ Adjusting for risk factors ${ }^{\mathrm{a}}$} \\
\hline & Nephropathy & All patients & Nephropathy & All patients \\
\hline $\begin{array}{l}\text { Telomere length as a continuous variable: } \\
\text { HR for } 1 \text { kilobase pair decrease }\end{array}$ & $1.53(1.12-2.08)$ & $1.49(1.23-1.98)$ & $1.15(0.83-1.58)$ & $1.15(0.87-1.54)$ \\
\hline$p$ value & 0.007 & 0.005 & 0.41 & 0.32 \\
\hline $\begin{array}{l}\text { Telomere length as a categorical variable: } \\
\text { HR for going from the longest tertile to the shortest tertile }\end{array}$ & $2.64(1.36-5.14)$ & $2.54(1.39-4.65)$ & $2.34(1.06-4.54)$ & $1.87(1.03-3.74)$ \\
\hline$p$ value & 0.004 & 0.002 & 0.02 & 0.04 \\
\hline
\end{tabular}

Values are HR $(95 \% \mathrm{CI})$ and $p$ value.

${ }^{\mathrm{a}}$ In the multivariate Cox regression analysis, the values are adjusted for age, sex, smoking, systolic blood pressure, total cholesterol, $\mathrm{Hb} \mathrm{A}_{1 \mathrm{c}}(\%)$, $\log$ urinary albumin excretion rate, previous myocardial infarction or apoplexia at baseline and telomere length as a continuous variable or a categorical variable (tertiles of telomere length).

0.45. Telomere length was significantly inversely correlated to age $(r=-0.33)$, systolic blood pressure $(r=-0.21)$, and duration of diabetes $(r=-0.31), p<0.01$. The predicting value of telomere length is shown in Table 1.

During follow-up, 75 patients died, including 63 of 157 patients with diabetic nephropathy (40\%). In the tertile with the shortest telomere length, 36 of 97 patients (37\%) died, and in the middle tertile 24 of 86 patients (28\%) of patients died, whereas in the tertile with the longest telomere length 15 of 90 patients (17\%) of patients died from any cause, log rank test $p=0.017$ (Fig. 1).

Telomere length was not related to end-stage renal disease, nor did we find any association between telomere length and rate of decline in GFR.

\section{Discussion}

In 273 type 1 diabetic patients, of which 157 had diabetic nephropathy, we found no difference in telomere length between patients with or without diabetic nephropathy. Telomere length related inversely to age, duration of diabetes and systolic blood pressure. Patients with shorter telomere length had increased mortality. When evaluating the predictive value of telomere length in a Cox regression model, we found that even after adjusting for traditional risk factors, telomere length was predictive of all-cause mortality. Telomere length was not associated with renal outcome.

To our knowledge, this is the first study to evaluate the long-term predictive value of telomere length in patients with type 1 diabetes with or without diabetic nephropathy. Previously, telomere shortening has been found in patients with type 1 diabetes [5] when compared with non-diabetic individuals. Some suggest that 'vascular ageing' gives telomere shortening. An association between arterial stiff- ness and telomere shortening was found in type 2 diabetic patients with microalbuminuria [6]. The authors conclude that microalbuminuric patients have more pronounced vascular ageing than normoalbuminuric controls and suggest that telomere shortening is a marker of this pronounced vascular ageing. We did not find our type 1 diabetic patients with diabetic nephropathy to have shorter telomere length than normoalbuminuric patients, even though patients with diabetic nephropathy had a much higher mortality. Recently, the authors of a sub-analysis from the Framingham study [7], suggested that an overactive renin angiotensin system may promote an increased burden of inflammation and oxidative stress, expressed as

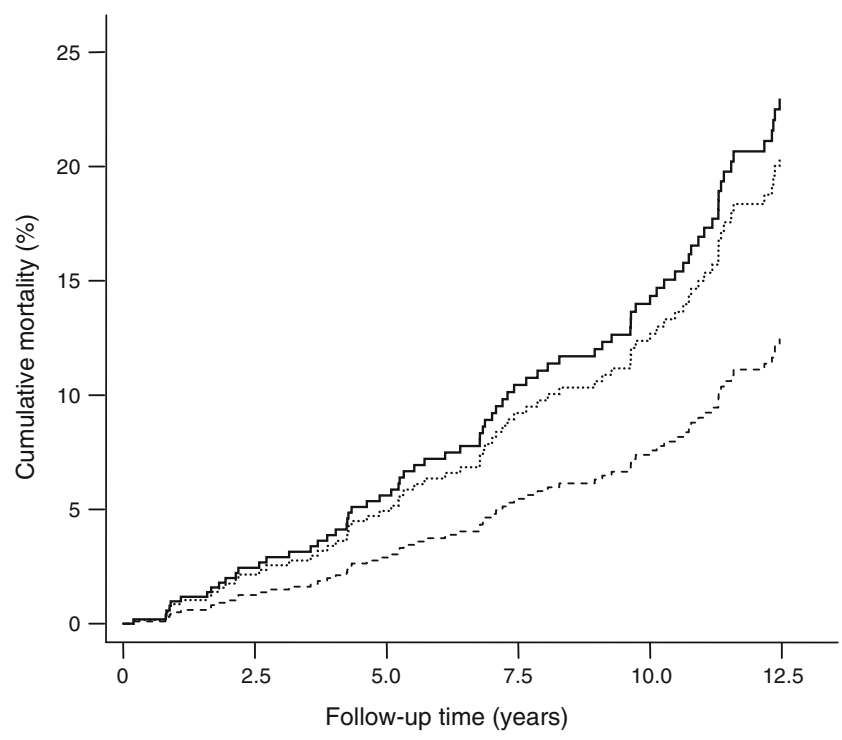

Fig. 1 Cumulative mortality for 273 patients with type 1 diabetes, divided into tertiles of telomere length (black line, shortest tertile; dotted line, middle tertile; dashed line, longest tertile). The graph is adjusted for age, sex, smoking, systolic blood pressure, total cholesterol, $\mathrm{HbA}_{1 \mathrm{c}}, \log$ urinary albumin excretion rate and previous myocardial infarction or apoplexia at baseline 
telomere shortening. Our patients with diabetic nephropathy did not have shorter telomere length than our normoalbuminuric controls. We did not have measures of components of the renin angiotensin system, but if telomere shortening was a result of an overactive renin angiotensin system we would expect to see relative shortening of telomeres in patients with diabetic nephropathy compared with normoalbuminuric patients.

Limitations to our study are the relative small sample size and the fact that the original design was to compare the two groups and only secondarily to analyse telomere length as a predictor of all-cause mortality. The determination of telomere length is a difficult analysis, and information on the coefficient of variation of telomere length could not be assessed in the current cohort.

The present study finds no difference in telomere length between patients with nephropathy and normoalbuminuria. As a secondary objective we found an independent relationship between telomere shortening and all-cause mortality. We found no associations between telomere shortening and progression of renal disease. Our data suggest that telomere shortening is in some way either involved in or influenced by pathological mechanisms, leading to poor prognosis.
Duality of interest The authors declare that there is no duality of interest associated with this manuscript.

\section{References}

1. Olovnikov AM (1973) A theory of marginotomy. The incomplete copying of template margin in enzymic synthesis of polynucleotides and biological significance of the phenomenon. J Theor Biol 41:181-190

2. von Zqlinicki $T$ (2000) Role of oxidative stress in telomere length regulation and replicative senescence. Ann N Y Acad Sci 908:99-110

3. Parving H-H, Andersen AR, Smidt UM, Svendsen PA (1983) Early aggressive antihypertensive treatment reduces rate of decline in kidney function in diabetic nephropathy. Lancet 1:1175-1179

4. Tarnow L, Cambien F, Rossing P et al (1995) Lack of relationship between an insertion/deletion polymorphism in the angiotensin-Iconverting enzyme gene and diabetic nephropathy and proliferative retinopathy in IDDM patients. Diabetes 44:489-494

5. Jeanclos E, Krolewski A, Skurnick J et al (1998) Shortened telomere length in white blood cells of patients with IDDM. Diabetes 47:482-486

6. Tentolouris N, Nzietchueng R, Cattan V et al (2007) White blood cells telomere length is shorter in males with type 2 diabetes and microalbuminuria. Diabetes Care 30:2909-2915

7. Vasan RS, Demissie S, Kimura M et al (2008) Association of leukocyte telomere length with circulating biomarkers of the reninangiotensin-aldosterone system: the Framingham Heart Study. Circulation 117:1138-1144 